九州大学学術情報リポジトリ

Kyushu University Institutional Repository

\title{
A regularization of a reaction-diffusion system approximation to the two-phase Stefan problem
}

Murakawa, Hidek i

Graduate School of Mathematics, Kyushu University

http://hdl. handle. net/2324/3378

出版情報 : MHF Preprint Series. 2005-34，2005-12-15. 九州大学大学院数理学研究院 バージョン：

権利関係 : 


\section{MHF Preprint Series}

Kyushu University

21st Century COE Program

Development of Dynamic Mathematics with High Functionality

\section{A regularization of a reaction-diffusion system approximation to the two-phase Stefan problem}

\section{H. Murakawa}

MHF 2005-34

( Received December 15, 2005 )

Faculty of Mathematics

Kyushu University

Fukuoka, JAPAN 


\title{
A regularization of a reaction-diffusion system approximation to the two-phase Stefan problem
}

\author{
Hideki Murakawa \\ Graduate School of Mathematics, Kyushu University, Fukuoka 812-8581, Japan
}

\begin{abstract}
Reaction-diffusion system approximations to the classical two-phase Stefan problem are considered in the present study. A reaction-diffusion system approximation to the Stefan problem has been proposed by Hilhorst et al. from an ecological point of view, and they have given convergence results for the system. In the present study, a new reaction-diffusion system approximation to the Stefan problem is proposed based on regularization of the enthalpy-temperature constitutive relation. The rates of convergence for each reaction-diffusion system are investigated in order to compare these two systems.
\end{abstract}

Key words: Stefan problem, reaction-diffusion systems, regularization, convergence rates

\section{Introduction}

Heat transfer problems involving phase change arise in a number of important physical and industrial contexts. A typical model of such problems, the classical two-phase Stefan problem, which describes the melting or freezing of a solid, in a rather simplified way, by accounting for the heat diffusion in each phase and the exchange of latent heat at the phase interface, is considered.

Let $\Omega \subset \mathbb{R}^{N}(N \in \mathbb{N})$ be a bounded domain with smooth boundary $\partial \Omega$. The domain $\Omega$ is divided into liquid and solid phases by unknown interface $\Gamma(t)$ at time $t \in(0, T)$, where $T$ is a positive constant. These phases are denoted by $\Omega_{u}(t)$ and $\Omega_{v}(t)$, respectively. Heat flow occurs in both the liquid and solid 
phases:

$$
\left\{\begin{array}{lll}
\frac{\partial u}{\partial t}=d_{1} \Delta u & \text { in } & \Omega_{u}:=\cup_{0<t<T} \Omega_{u}(t) \times\{t\}, \\
\frac{\partial v}{\partial t}=d_{2} \Delta v & \text { in } & \Omega_{v}:=\cup_{0<t<T} \Omega_{v}(t) \times\{t\},
\end{array}\right.
$$

where $d_{1}$ and $d_{2}$ are given diffusion coefficients. The functions $u>0$ and $-v<0$ represent temperatures of the liquid and solid, respectively. Here, the melting temperature is taken to be zero. In addition, temperature is zero on the interface:

$$
u=v=0 \quad \text { on } \quad \Gamma:=\cup_{0<t<T} \Gamma(t) \times\{t\} .
$$

The energy balance between the two phases leads to the following Stefan condition:

$$
\lambda V_{n}=-d_{1} \frac{\partial u}{\partial n}-d_{2} \frac{\partial v}{\partial n} \quad \text { on } \quad \Gamma,
$$

where $\lambda$ is the latent heat coefficient that represents the latent heat of crystallization per unit mass, $n$ is the unit normal vector on $\Gamma(t)$ oriented from $\Omega_{u}(t)$ to $\Omega_{v}(t)$, and $V_{n}$ is the normal speed of the interface. Equations (1)-(3) constitute the classical formulation of the classical two-phase Stefan problem.

The Stefan problem can be interpreted as an ecological problem. The problem can be stated as a free boundary problem for two competing species that are regionally segregated. Functions $u$ and $v$ are the densities of the competing species, which move by the diffusions described by (1). The regions $\Omega_{u}$ and $\Omega_{v}$ denote the habitats for $u$ and $v$, respectively. The condition (2) shows that the regional segregation occurs for two competing species. The Stefan condition (3) is not described herein. Although the two species are completely segregated in this problem, the competition between the two species is regarded as being very strong. This leads to a reaction-diffusion system with a sufficiently small parameter $\varepsilon$, as follows:

$$
\left\{\begin{array}{l}
\frac{\partial u}{\partial t}=d_{1} \Delta u-\frac{1}{\varepsilon} u v \quad \text { in } \quad Q:=\Omega \times(0, T), \\
\frac{\partial v}{\partial t}=d_{2} \Delta v-\frac{1}{\varepsilon} u v \quad \text { in } \quad Q .
\end{array}\right.
$$

The two species coexist everywhere in $\Omega$, while $\varepsilon$ is a positive constant. Then, the following question arises: Is there any relation between (4) and the Stefan problem? Dancer et al. have answered the question $[3,4]$. In a sense, the system (4) is similar to the Stefan problem without the latent heat. In this case, the Stefan condition (3) is replaced by

$$
0=-d_{1} \frac{\partial u}{\partial n}-d_{2} \frac{\partial v}{\partial n} \quad \text { on } \quad \Gamma .
$$

Because the latent heat coefficient is zero in this case, the following question arises: Are there any reaction diffusion system approximations to the Stefan 
problem with positive latent heat? To answer this question, Hilhorst et al. considered the following reaction-diffusion system $[7,8]$ :

$$
\begin{cases}\frac{\partial u}{\partial t}=d_{1} \Delta u-\frac{1}{\varepsilon}(u v+\lambda p u) & \text { in } \quad Q \\ \frac{\partial v}{\partial t}=d_{2} \Delta v-\frac{1}{\varepsilon}(u v+\lambda v w) & \text { in } \quad Q \\ \frac{\partial w}{\partial t}=\frac{1}{\varepsilon}(p u-v w) & \text { in } \quad Q \\ \frac{\partial p}{\partial t}=\frac{1}{\varepsilon}(-p u+v w) & \text { in } \quad Q\end{cases}
$$

with the initial data satisfying

$$
w_{0}(x)=\left\{\begin{array}{lll}
1 & \text { if } & u_{0}(x)>0, \\
0 & \text { if } & u_{0}(x)=0,
\end{array} \quad p_{0}(x)=\left\{\begin{array}{lll}
1 & \text { if } & v_{0}(x)>0 \\
0 & \text { if } & v_{0}(x)=0
\end{array}\right.\right.
$$

for $x \in \Omega$ and

$$
w_{0}+p_{0}=1 \quad \text { a.e. in } \Omega .
$$

The initial data $w_{0}$ and $p_{0}$ are the characteristic functions of the initial habitats for $u$ and $v$, respectively. The relation (5) indicates that the initial distributions of $u$ and $v$ are completely segregated, and the initial interface $\Gamma(0)$ is a hypersurface. This system is interpreted ecologically as follows. Functions $u$ and $v$ represent the densities of two competing species that move by diffusion, and $w$ and $p$ are the characteristic-like functions of the habitats $\Omega_{u}$ and $\Omega_{v}$, respectively. The constant $\lambda$ denotes the cost rate when species $u$ attacks the habitat of species $v$, and vice versa. There are two different types of interactions between $u$ and $v$ in this problem. One is direct competitive interaction $(u v)$, and the other type is conflict type interactions ( $\lambda p u$ and $\lambda w v)$, where species $u(v)$ tries to invade the habitat of species $v(u)$, or species $v(u)$ tries to prevent species $u(v)$ from invading their own habitat.

Note that $(w+p)_{t}=0$, that is,

$$
w(x, t)+p(x, t)=w_{0}(x)+p_{0}(x)=1
$$

Function $\lambda w$ is transformed into $\tilde{w}$, which is denoted as $w$ again, and $\lambda p$ is transformed into $\tilde{p}$. The initial data $u_{0}, v_{0}$ and $w_{0}$ are approximated by $u_{0}^{\varepsilon}, v_{0}^{\varepsilon}$ and $w_{0}^{\varepsilon}$, respectively. In this paper, the homogeneous Neumann boundary conditions are assumed. The problem then yields the following reaction-diffusion 
system:

$$
(\mathrm{RD})_{0} \begin{cases}\frac{\partial u}{\partial t}=d_{1} \Delta u-\frac{1}{\varepsilon}(u v+(\lambda-w) u) & \text { in } \quad Q \\ \frac{\partial v}{\partial t}=d_{2} \Delta v-\frac{1}{\varepsilon}(u v+v w) & \text { in } Q \\ \frac{\partial w}{\partial t}=\quad \frac{1}{\varepsilon}((\lambda-w) u-v w) & \text { in } Q, \\ \frac{\partial u}{\partial \nu}=\frac{\partial v}{\partial \nu}=0 & \text { on } \partial \Omega \times(0, T), \\ u(\cdot, 0)=u_{0}^{\varepsilon}, v(\cdot, 0)=v_{0}^{\varepsilon}, w(\cdot, 0)=w_{0}^{\varepsilon} & \text { in } \Omega,\end{cases}
$$

where $\nu$ is the outward normal unit vector to the boundary $\partial \Omega$. Hereafter, the following assumptions are imposed on the initial data:

$$
\left\{\begin{array}{l}
u_{0}^{\varepsilon}, v_{0}^{\varepsilon} \in C(\bar{\Omega}), \quad w_{0}^{\varepsilon} \in L^{\infty}(\Omega), \\
0 \leq u_{0}^{\varepsilon} \leq M, \quad 0 \leq v_{0}^{\varepsilon} \leq M, \quad 0 \leq w_{0}^{\varepsilon} \leq \lambda \quad \text { in } \quad \Omega
\end{array}\right.
$$

for some positive constant $M$ independent of $\varepsilon$. Hilhorst et al. $[7,8]$ studied a number of relations between $(\mathrm{RD})_{0}$ and the Stefan problem. In stating their results for $(\mathrm{RD})_{0}$, another formulation of the classical two-phase Stefan problem in terms of enthalpy, which is the sum of sensible and latent heats, should be introduced.

The enthalpy formulation of the classical two-phase Stefan problem can be formulated as follows:

$$
(\mathrm{SP})\left\{\begin{array}{lll}
z_{t}=\Delta d(\phi(z)) & \text { in } \quad & Q \\
\frac{\partial d(\phi(z))}{\partial \nu}=0 & \text { on } & \partial \Omega \times(0, T) \\
z(x, 0)=z_{0}(x) & \text { for } & x \in \Omega
\end{array}\right.
$$

where $z_{0}$ is a given function, and the functions $d$ and $\phi$ are defined as

$$
d(r)=\left\{\begin{array}{l}
d_{1} r \text { if } r \geq 0, \\
d_{2} r \text { if } r<0,
\end{array} \quad \phi(r)= \begin{cases}r-\lambda & \text { if } r>\lambda, \\
0 & \text { if } 0 \leq r \leq \lambda, \\
r & \text { if } r<0\end{cases}\right.
$$

for $r \in \mathbb{R}$. Functions $z$ and $\phi(z)$ represent, physically, the enthalpy and the temperature, respectively. In the solid phase, $\phi(z)<0$, and in the liquid phase, $\phi(z)>0$. These two phases are separated by the zero level set of $\phi(z)$. In this formulation, the interface disappears as an explicit unknown. For example, the interface is recovered as the zero level set of the temperature $\phi(z)$. Next, the functions $u:=\phi_{1}(z):=\phi(z)^{+}, v:=\phi_{2}(z):=\phi(z)^{-}$and $w:=\phi_{3}(z):=z-\phi(z)$, are defined, where $\phi^{+}$indicates the positive part of $\phi$ and $\phi^{-}$indicates the 
negative part of $\phi$. Then, $u,-v$ and $w$ represent, physically, the temperature of the liquid, the temperature of the solid and the latent heat. Or, for an ecological system, $u, v$ and $w$ represent the densities of the two competing species and the characteristic-like function of the habitat for $u$, respectively.

This problem should be understood in a weak sense.

Definition 1 A function $z \in L^{\infty}\left(Q_{T}\right)$ is a weak solution of (SP) with an initial datum $z_{0} \in L^{\infty}(\Omega)$ if it satisfies $d(\phi(z)) \in L^{2}\left(0, T ; H^{1}(\Omega)\right)$ and

$$
\int_{0}^{T}\left\langle z, \zeta_{t}\right\rangle+\left\langle z_{0}, \zeta(\cdot, 0)\right\rangle=\int_{0}^{T}\langle\nabla d(\phi(z)), \nabla \zeta\rangle
$$

for all functions $\zeta \in \mathcal{K}:=\left\{\zeta \in H^{1}(Q) \mid \zeta(\cdot, T)=0\right\}$. Here, $\langle\cdot, \cdot\rangle$ denotes the inner product of $L^{2}(\Omega)$.

The unique existence of the weak solution of the problem and a relation between the classical form and the enthalpy form are known (see for example $[2,5,7,9,12]$ and the references therein).

The results for $(\mathrm{RD})_{0}$ reported by Hilhorst et al. can now be discussed.

Theorem 2 (Hilhorst, Iida, Mimura \& Ninomiya $[7,8]$ ) Suppose the initial data satisfy (6). Then, there exists a unique solution $\left(u^{\varepsilon}, v^{\varepsilon}, w^{\varepsilon}\right)$ of $(\mathrm{RD})_{0}$ in $Q$.

Let $z$ be a unique weak solution of (SP) with an initial datum $z_{0} \in L^{\infty}(\Omega)$. Assume that

$$
u_{0}^{\varepsilon} \rightarrow \phi_{1}\left(z_{0}\right), v_{0}^{\varepsilon} \rightarrow \phi_{2}\left(z_{0}\right), w_{0}^{\varepsilon} \rightarrow \phi_{3}\left(z_{0}\right) \quad \text { weakly in } L^{2}(\Omega) \text { as } \varepsilon \rightarrow 0
$$

and (6). Then,

$$
\begin{aligned}
& \left.\begin{array}{l}
u^{\varepsilon} \rightarrow \phi_{1}(z) \\
v^{\varepsilon} \rightarrow \phi_{2}(z)
\end{array}\right\} \text { strongly in } L^{2}(Q) \text { and weakly in } L^{2}\left(0, T ; H^{1}(\Omega)\right), \\
& z^{\varepsilon} \rightarrow z \quad \text { weakly in } L^{2}(Q)
\end{aligned}
$$

as $\varepsilon \rightarrow 0$. Here, $z^{\varepsilon}:=u^{\varepsilon}-v^{\varepsilon}+w^{\varepsilon}$.

Thus, Hilhorst et al. have given a reaction diffusion system approximation to the Stefan problem from an ecological point of view and have proved the above convergence results.

The diffusion vanishes in (SP), where $z \in(0, \lambda)$. Vanishing diffusion characterizes the presence of a free boundary $\Gamma$, and the solution exhibits a lack of regularity across $\Gamma$. Thus, the solution of the Stefan problem generally has low regularity properties. Consequently, regularizations of the enthalpytemperature constitutive relation are sometimes used for the Stefan problem 
(see for example $[1,6,14,15])$. Next, the combined use of a reaction diffusion system approximation with a regularization procedure is considered. Then, a new reaction-diffusion system $(\mathrm{RD})_{\xi}$ is proposed (see the following section) that approximates the Stefan problem. Under certain assumptions, convergence results similar to Theorem 2 can be obtained for $(\mathrm{RD})_{\xi}$. However, the reaction-diffusion system $(\mathrm{RD})_{\xi}$ is expected to give a better approximation than $(\mathrm{RD})_{0}$. Therefore, rates of convergence with respect to $\varepsilon$ are investigated for each reaction-diffusion system.

In the next section, a reaction-diffusion system and the theoretical results for the rates of convergence of the system $(\mathrm{RD})_{0}$ and those of the proposed system are presented. In Sections 3 and 4, the results for the system (RD) $)_{0}$ and the proposed system, respectively, are presented.

\section{A reaction-diffusion system and main results}

The following reaction-diffusion system with sufficiently small parameter $\varepsilon$ and $\xi$ is proposed:

$$
(\mathrm{RD})_{\xi} \begin{cases}\frac{\partial u}{\partial t}=d_{1} \Delta u-\frac{1}{\varepsilon}(u v+(\eta-w) u) & \text { in } \quad Q, \\ \frac{\partial v}{\partial t}=d_{2} \Delta v-\frac{1}{\varepsilon}(u v+v w) & \text { in } \quad Q, \\ \frac{\partial w}{\partial t}=\xi \Delta w+\frac{1}{\varepsilon}((\eta-w) u-v w) & \text { in } \quad Q, \\ \frac{\partial u}{\partial \nu}=\frac{\partial v}{\partial \nu}=\frac{\partial w}{\partial \nu}=0 & \text { on } \quad \partial \Omega \times(0, T), \\ u(\cdot, 0)=u_{0}^{\varepsilon, \xi}, v(\cdot, 0)=v_{0}^{\varepsilon, \xi}, w(\cdot, 0)=w_{0}^{\varepsilon, \xi} & \text { in } \quad \Omega,\end{cases}
$$

where $\eta=d_{1} \lambda /\left(d_{1}-\xi\right)$.

When $\xi$ is zero, this system is obviously reduced to the system (RD) $)_{0}$. That is, the system $(\mathrm{RD})_{\xi}$ is an extension of $(\mathrm{RD})_{0}$.

For fixed $\xi$, the system $(\mathrm{RD})_{0}$ can be regarded as an approximation to a weak solution of equation

$$
\begin{cases}\frac{\partial z_{\xi}}{\partial t}=\Delta \beta_{\xi}\left(z_{\xi}\right), & \text { in } \quad Q \\ \frac{\partial \beta_{\xi}\left(z_{\xi}\right)}{\partial \nu}=0 & \text { on } \quad \partial \Omega \times(0, T) \\ z_{\xi}(x, 0)=z_{\xi 0}(x) & \text { for } \quad x \in \Omega\end{cases}
$$


Fig. 1. Constitutive relation $\beta$ and regularized relation $\beta_{\xi}$.
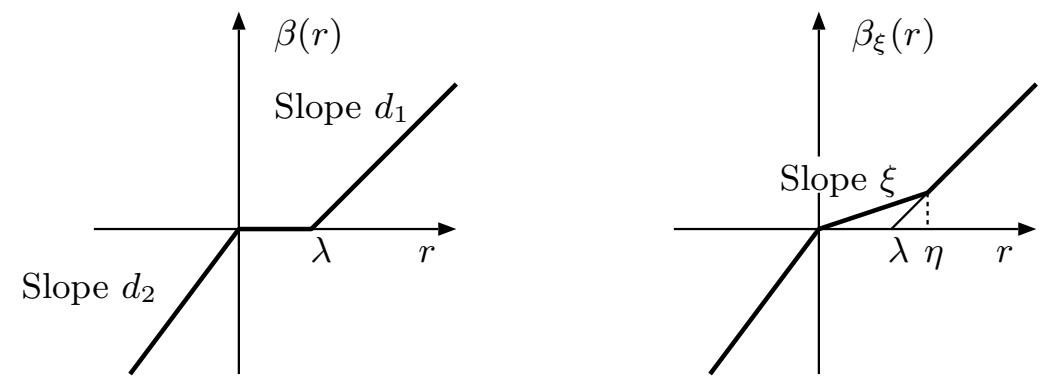

where $z_{\xi 0} \in C(\bar{\Omega}), \beta_{\xi}$ is defined as (see Figure 1)

$$
\beta_{\xi}(r)= \begin{cases}d_{1}(r-\lambda) & \text { if } r>\eta, \\ \xi r & \text { if } 0 \leq r \leq \eta \\ d_{2} r & \text { if } r<0 .\end{cases}
$$

The weak solution of (8) is Hölder continuous in $\bar{Q}$ (see [10]), although the weak solution of $(\mathrm{SP})$ is generally discontinuous. In this sense, $(\mathrm{RD})_{\xi}$ can be interpreted as the combination of a reaction diffusion system approximation and a regularization procedure.

The following assumption is made regarding the initial data:

$$
\left\{\begin{array}{l}
u_{0}^{\varepsilon, \xi}, v_{0}^{\varepsilon, \xi}, w_{0}^{\varepsilon, \xi} \in C(\bar{\Omega}), \\
0 \leq u_{0}^{\varepsilon, \xi} \leq M, \quad 0 \leq v_{0}^{\varepsilon, \xi} \leq M, \quad 0 \leq w_{0}^{\varepsilon, \xi} \leq \eta \quad \text { in } \quad \Omega
\end{array}\right.
$$

for some positive constant $M$ independent of $\varepsilon$ and $\xi$.

Then, the existence and uniqueness of the solution of $(\mathrm{RD})_{\xi}$ follows from Lunardi [11, Proposition 7.3.2].

Next, the results for the convergence rates of reaction-diffusion systems $(\mathrm{RD})_{\xi}$, $\xi \geq 0$ are presented.

Theorem 3 Let $z$ be a unique weak solution of (SP) with an initial datum $z_{0} \in$ $L^{\infty}(\Omega)$, and let $\left(u^{\varepsilon}, v^{\varepsilon}, w^{\varepsilon}\right)$ be a unique solution of $(\mathrm{RD})_{0}$ with the initial data satisfying assumption (6). Then, there exists a positive constant $C$ independent of $\varepsilon$ such that

$$
\begin{array}{r}
\left\|u-u^{\varepsilon}\right\|_{L^{2}(Q)}+\left\|v-v^{\varepsilon}\right\|_{L^{2}(Q)}+\left\|\int_{0}^{t}\left(\theta-\theta^{\varepsilon}\right)\right\|_{L^{\infty}\left(0, T ; H^{1}(\Omega)\right)} \\
+\left\|z-z^{\varepsilon}\right\|_{L^{\infty}\left(0, T ;\left(H^{1}(\Omega)\right)^{*}\right)} \leq C\left(\varepsilon^{1 / 2}+\sigma(\varepsilon)\right)^{1 / 2},
\end{array}
$$

where $u:=\phi_{1}(z), v:=\phi_{2}(z), \theta:=\beta(z):=d(\phi(z)), \theta^{\varepsilon}:=d_{1} u^{\varepsilon}-d_{2} v^{\varepsilon}$, $z^{\varepsilon}:=u^{\varepsilon}-v^{\varepsilon}+w^{\varepsilon}, \sigma(\varepsilon):=\left\|z_{0}-z_{0}^{\varepsilon}\right\|_{L^{2}(\Omega)}^{2}$ and $z_{0}^{\varepsilon}=u_{0}^{\varepsilon}-v_{0}^{\varepsilon}+w_{0}^{\varepsilon}$. 
Fig. 2. Functions $\phi_{1}^{\xi}, \phi_{2}$ and $\phi_{3}^{\xi}$.
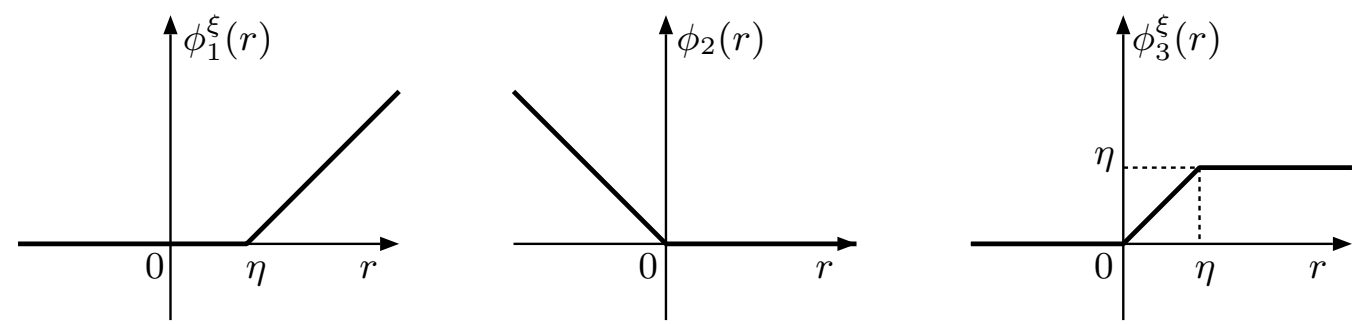

The rate of convergence is $\mathcal{O}\left(\varepsilon^{1 / 4}\right)$ if $\sigma$ is of order $\varepsilon^{1 / 2}$.

Theorem 4 Let $z$ be a unique weak solution of (SP) with an initial datum $z_{0} \in L^{\infty}(\Omega)$, and let $\left(u_{\xi}^{\varepsilon}, v_{\xi}^{\varepsilon}, w_{\xi}^{\varepsilon}\right)$ be a unique solution of $(\mathrm{RD})_{\xi}$ with initial data satisfying the assumption given in (9). Then, there exists a positive constant $C$ independent of $\varepsilon$ and $\xi$ such that

$$
\begin{aligned}
& \left\|u-u_{\xi}^{\varepsilon}\right\|_{L^{2}(Q)}+\left\|v-v_{\xi}^{\varepsilon}\right\|_{L^{2}(Q)}+\xi^{1 / 2}\left\|w-w_{\xi}^{\varepsilon}\right\|_{L^{2}(Q)} \\
& \quad+\left\|\int_{0}^{t}\left(\theta-\theta_{\xi}^{\varepsilon}\right)\right\|_{L^{\infty}\left(0, T ; H^{1}(\Omega)\right)}+\left\|z-z_{\xi}^{\varepsilon}\right\|_{L^{\infty}\left(0, T ;\left(H^{1}(\Omega)\right)^{*}\right)} \\
& \quad \leq C\left(\xi\left|A_{\xi}(z)\right|+\xi^{2}+\frac{\varepsilon}{\xi}+\sigma(\varepsilon, \xi)\right)^{1 / 2}
\end{aligned}
$$

where $u=\phi_{1}(z), v=\phi_{2}(z), w=\phi_{3}(z), \theta=\beta(z), \theta_{\xi}^{\varepsilon}:=d_{1} u_{\xi}^{\varepsilon}-d_{2} v_{\xi}^{\varepsilon}+\xi w_{\xi}^{\varepsilon}$, $z_{\xi}^{\varepsilon}:=u_{\xi}^{\varepsilon}-v_{\xi}^{\varepsilon}+w_{\xi}^{\varepsilon}, \sigma(\varepsilon, \xi):=\left\|z_{0}-z_{0}^{\varepsilon, \xi}\right\|_{L^{2}(\Omega)}^{2}, z_{0}^{\varepsilon, \xi}:=u_{0}^{\varepsilon, \xi}-v_{0}^{\varepsilon, \xi}+w_{0}^{\varepsilon, \xi}$ and $A_{\xi}(z):=\{(x, t) \in Q \mid 0 \leq \beta(z(x, t)) \leq \eta \xi\}$.

The general rate of convergence is $\mathcal{O}\left(\varepsilon^{1 / 4}\right)$ if $\xi$ and $\sigma$ are chosen to be of order $\varepsilon^{1 / 2}$. However, if the non-degeneracy property $\left|A_{\xi}(z)\right| \leq C \xi$ for some positive constant $C$ is valid, as investigated by Nochetto [15], and if $\xi$ is chosen to be of order $\varepsilon^{1 / 3}$ and $\sigma$ is of order $\varepsilon^{2 / 3}$, then the rate becomes $\mathcal{O}\left(\varepsilon^{1 / 3}\right)$. In addition, the corresponding latent heat $w_{\xi}^{\varepsilon}$ and the corresponding enthalpy $z_{\xi}^{\varepsilon}$ converge strongly in $L^{2}(Q)$. The rate of convergence is $\mathcal{O}\left(\varepsilon^{1 / 6}\right)$. Thus, better results are obtained for the reaction-diffusion system $(\mathrm{RD})_{\xi}$ than for $(\mathrm{RD})_{0}$.

The relationship between $(\mathrm{RD})_{\xi}$ and $(8)$ for fixed $\xi>0$ can be obtained as follows. Let functions $\phi_{i}^{\xi}(i=1,3)$ be defined as follows (see Figure 2):

$$
\phi_{1}^{\xi}(r)=\left\{\begin{array}{ll}
r-\eta & \text { if } r \geq \eta, \\
0 & \text { if } r<\eta,
\end{array} \quad \phi_{3}^{\xi}(r)= \begin{cases}\eta & \text { if } r \geq \eta, \\
r & \text { if } 0<r<\eta, \\
0 & \text { if } r \leq 0\end{cases}\right.
$$

for $r \in \mathbb{R}$. Note that the relation $\beta_{\xi}=d_{1} \phi_{1}^{\xi}-d_{2} \phi_{2}+\xi \phi_{3}^{\xi}$ holds.

Corollary 5 Let $z_{\xi}$ be a unique weak solution of (8) with an initial datum 
$z_{0} \in L^{\infty}(\Omega)$, and let $\left(u_{\xi}^{\varepsilon}, v_{\xi}^{\varepsilon}, w_{\xi}^{\varepsilon}\right)$ be a unique solution of $(\mathrm{RD})_{\xi}$ with initial data satisfying the assumption give in (9). Then, there exists a positive constant $C$ independent of $\varepsilon$ and $\xi$ such that

$$
\begin{aligned}
& \left\|u_{\xi}-u_{\xi}^{\varepsilon}\right\|_{L^{2}(Q)}+\left\|v_{\xi}-v_{\xi}^{\varepsilon}\right\|_{L^{2}(Q)}+\left\|w_{\xi}-w_{\xi}^{\varepsilon}\right\|_{L^{2}(Q)} \\
& \quad+\left\|\int_{0}^{t}\left(\theta_{\xi}-\theta_{\xi}^{\varepsilon}\right)\right\|_{L^{\infty}\left(0, T ; H^{1}(\Omega)\right)}+\left\|z_{\xi}-z_{\xi}^{\varepsilon}\right\|_{L^{\infty}\left(0, T ;\left(H^{1}(\Omega)\right)^{*}\right)} \\
& \quad \leq C(\varepsilon+\sigma(\varepsilon))^{1 / 2},
\end{aligned}
$$

where $u_{\xi}=\phi_{1}^{\xi}\left(z_{\xi}\right), v_{\xi}=\phi_{2}\left(z_{\xi}\right), w_{\xi}=\phi_{3}^{\xi}\left(z_{\xi}\right), \theta_{\xi}=\beta_{\xi}\left(z_{\xi}\right), \theta_{\xi}^{\varepsilon}:=d_{1} u_{\xi}^{\varepsilon}-d_{2} v_{\xi}^{\varepsilon}+$ $\xi w_{\xi}^{\varepsilon}, z_{\xi}^{\varepsilon}:=u_{\xi}^{\varepsilon}-v_{\xi}^{\varepsilon}+w_{\xi}^{\varepsilon}, \sigma(\varepsilon):=\left\|z_{0}-z_{0}^{\varepsilon, \xi}\right\|_{L^{2}(\Omega)}^{2}$, and $z_{0}^{\varepsilon, \xi}:=u_{0}^{\varepsilon, \xi}-v_{0}^{\varepsilon, \xi}+w_{0}^{\varepsilon, \xi}$.

The rate of convergence is $\mathcal{O}\left(\varepsilon^{1 / 2}\right)$ if $\sigma$ is of order $\varepsilon$. Corollary 5 also implies that the solution of (4) converges to the weak solution of the Stefan problem without the latent heat and that its rate of convergence in $L^{2}(Q)$ is $\mathcal{O}\left(\varepsilon^{1 / 2}\right)$.

\section{$3 \quad$ A proof of Theorem 3}

In this section, a proof of Theorem 3 is given. To this end, a number of lemmas are presented for the functions $u^{\varepsilon}, v^{\varepsilon}$ and $w^{\varepsilon}$.

Lemma 6 The functions $u^{\varepsilon}, v^{\varepsilon}$ and $w^{\varepsilon}$ are uniformly bounded with respect to $\varepsilon$ in $L^{\infty}(Q)$. More precisely, the functions $u^{\varepsilon}, v^{\varepsilon}$ and $w^{\varepsilon}$ satisfy

$$
0 \leq u^{\varepsilon} \leq M, \quad 0 \leq v^{\varepsilon} \leq M, \quad 0 \leq w^{\varepsilon} \leq \lambda \quad \text { in } \quad Q
$$

Proof. The assertion follows from the maximum principle.

Lemma 7 There exists a positive constant $C$ independent of $\varepsilon$ such that

$$
\int_{0}^{T}\left\{\left\langle u^{\varepsilon}, v^{\varepsilon}\right\rangle+\left\langle\lambda-w^{\varepsilon}, u^{\varepsilon}\right\rangle+\left\langle v^{\varepsilon}, w^{\varepsilon}\right\rangle\right\} \leq C \varepsilon .
$$

Proof. Integration of the equation for $u^{\varepsilon}$ in $Q$ yields

$$
\iint_{Q} \frac{1}{\varepsilon}\left(u^{\varepsilon} v^{\varepsilon}+\left(\lambda-w^{\varepsilon}\right) u^{\varepsilon}\right)=\int_{\Omega}\left(u_{0}^{\varepsilon}-u^{\varepsilon}(\cdot, T)\right) \leq M|\Omega|,
$$

which implies the first and second terms of the desired estimate. Similarly, the third term can be shown by integrating the equation for $v^{\varepsilon}$. 
Lemma 8 There exists a positive constant $C$ independent of $\varepsilon$ such that

$$
d_{1}\left\|\nabla u^{\varepsilon}\right\|_{L^{2}(Q)}+d_{2}\left\|\nabla v^{\varepsilon}\right\|_{L^{2}(Q)} \leq C .
$$

Proof. Multiplying the equation for $u^{\varepsilon}$ by $u^{\varepsilon}$ and integrating by parts on $\Omega$, we have

$$
\frac{1}{2} \frac{d}{d t} \int_{\Omega}\left(u^{\varepsilon}\right)^{2}+d_{1} \int_{\Omega}\left|\nabla u^{\varepsilon}\right|^{2}+\int_{\Omega} \frac{1}{\varepsilon}\left(\left(u^{\varepsilon}\right)^{2} v^{\varepsilon}+\left(\lambda-w^{\varepsilon}\right)\left(u^{\varepsilon}\right)^{2}\right)=0 .
$$

Integrating on $(0, T)$ and using Lemmas 6 and 7 , we can obtain the first term. The second term can be similarly obtained.

Lemmas 6 and 8 imply that the functions $u^{\varepsilon}$ and $v^{\varepsilon}$ are uniformly bounded with respect to $\varepsilon$ in $L^{2}\left(0, T ; H^{1}(\Omega)\right)$.

From $(\mathrm{RD})_{0}$, we deduce that

$$
\frac{\partial z^{\varepsilon}}{\partial t}=\Delta \theta^{\varepsilon}
$$

Multiplying this expression by a test function $\zeta \in \mathcal{K}$ and integrating by parts, we obtain the identity

$$
\int_{0}^{T}\left\langle z^{\varepsilon}, \zeta_{t}\right\rangle+\left\langle z_{0}^{\varepsilon}, \zeta(\cdot, 0)\right\rangle=\int_{0}^{T}\left\langle\nabla \theta^{\varepsilon}, \nabla \zeta\right\rangle
$$

After subtraction from (10) to (7), we obtain the relation

$$
-\int_{0}^{T}\left\langle e_{z}, \zeta_{t}\right\rangle-\left\langle e_{z_{0}}, \zeta(\cdot, 0)\right\rangle+\int_{0}^{T}\left\langle\nabla e_{\theta}, \nabla \zeta\right\rangle=0
$$

for all $\zeta \in \mathcal{K}$. Here, errors are defined as

$$
e_{z}:=z-z^{\varepsilon}, \quad e_{\theta}:=\theta-\theta^{\varepsilon}, \quad e_{z_{0}}:=z_{0}-z_{0}^{\varepsilon} .
$$

The concept behind the proof is to use a stable test function in (11).

Proof of Theorem 3. For fixed $t_{0} \in(0, T]$, the following test function is proposed:

$$
\zeta(x, t)= \begin{cases}\int_{t}^{t_{0}} e_{\theta}(x, s) d s & \text { if } 0 \leq t<t_{0} \\ 0 & \text { if } t_{0} \leq t \leq T\end{cases}
$$


The first term of the left-hand side of (11) yields

$$
-\int_{0}^{T}\left\langle e_{z}, \zeta_{t}\right\rangle=\int_{0}^{t_{0}}\left\langle e_{z}, e_{\theta}\right\rangle
$$

The term $\left\langle e_{z}, e_{\theta}\right\rangle$ is then estimated. For a.e. $(x, t) \in Q$,

$$
\begin{aligned}
\left\langle e_{z}, e_{\theta}\right\rangle & =\left\langle e_{z}, \theta-\beta\left(z^{\varepsilon}\right)\right\rangle+\left\langle e_{z}, \beta\left(z^{\varepsilon}\right)-\theta^{\varepsilon}\right\rangle \\
& =d_{1}\left\langle e_{z}, u-\phi_{1}\left(z^{\varepsilon}\right)\right\rangle-d_{2}\left\langle e_{z}, v-\phi_{2}\left(z^{\varepsilon}\right)\right\rangle+\left\langle e_{z}, \beta\left(z^{\varepsilon}\right)-\theta^{\varepsilon}\right\rangle .
\end{aligned}
$$

The first term is estimated by means of the definition of $\phi_{1}$; namely

$$
\begin{array}{r}
d_{1}\left\langle z-z^{\varepsilon}, \phi_{1}(z)-\phi_{1}\left(z^{\varepsilon}\right)\right\rangle \geq d_{1}\left\|\phi_{1}(z)-\phi_{1}\left(z^{\varepsilon}\right)\right\|_{L^{2}(\Omega)}^{2} \\
\geq d_{1}\left(\frac{1}{2}\left\|e_{u}\right\|_{L^{2}(\Omega)}^{2}-\left\|\phi_{1}\left(z^{\varepsilon}\right)-u^{\varepsilon}\right\|_{L^{2}(\Omega)}^{2}\right),
\end{array}
$$

where $e_{u}:=u-u^{\varepsilon}$. In the same way, we obtain

$$
-d_{2}\left\langle z-z^{\varepsilon}, \phi_{2}(z)-\phi_{2}\left(z^{\varepsilon}\right)\right\rangle \geq d_{2}\left(\frac{1}{2}\left\|e_{v}\right\|_{L^{2}(\Omega)}^{2}-\left\|\phi_{2}\left(z^{\varepsilon}\right)-v^{\varepsilon}\right\|_{L^{2}(\Omega)}^{2}\right) .
$$

Here, $e_{v}:=v-v^{\varepsilon}$. Using the Cauchy-Schwarz inequality, we obtain

$$
\left\langle e_{z}, \beta\left(z^{\varepsilon}\right)-\theta^{\varepsilon}\right\rangle \leq\left\|e_{z}\right\|_{L^{2}(\Omega)}\left\|\beta\left(z^{\varepsilon}\right)-\theta^{\varepsilon}\right\|_{L^{2}(\Omega)} .
$$

The inequalities for $u, v \geq 0$ and $w \in[0, \lambda]$ are easily obtained:

$$
\left\{\begin{array}{l}
\left|u-\phi_{1}(u-v+w)\right|^{2} \leq u v+(\lambda-w) u \\
\left|v-\phi_{2}(u-v+w)\right|^{2} \leq u v+v w \\
\left|w-\phi_{3}(u-v+w)\right|^{2} \leq v w+(\lambda-w) u
\end{array}\right.
$$

It follows from Lemma 7 and (12) that

$$
\left\|\phi_{1}\left(z^{\varepsilon}\right)-u^{\varepsilon}\right\|_{L^{2}(Q)}^{2}+\left\|\phi_{2}\left(z^{\varepsilon}\right)-v^{\varepsilon}\right\|_{L^{2}(Q)}^{2}+\left\|\beta\left(z^{\varepsilon}\right)-\theta^{\varepsilon}\right\|_{L^{2}(Q)}^{2} \leq C \varepsilon,
$$

where $C$ is a positive constant that is independent of $\varepsilon$.

From the elementary relation

$$
2 a b \leq \alpha a^{2}+\frac{1}{\alpha} b^{2}
$$

for $a, b \in \mathbb{R}$ and $\alpha>0$, we have

$$
\begin{aligned}
\left\langle e_{z_{0}}, \zeta(\cdot, 0)\right\rangle & =\int_{0}^{t_{0}}\left\langle e_{z_{0}}, e_{\theta}\right\rangle=d_{1} \int_{0}^{t_{0}}\left\langle e_{z_{0}}, e_{u}\right\rangle-d_{2} \int_{0}^{t_{0}}\left\langle e_{z_{0}}, e_{v}\right\rangle \\
& \leq\left(d_{1}+d_{2}\right) T\left\|e_{z_{0}}\right\|_{L^{2}(\Omega)}^{2}+\frac{d_{1}}{4} \int_{0}^{t_{0}}\left\|e_{u}\right\|_{L^{2}(\Omega)}^{2}+\frac{d_{2}}{4} \int_{0}^{t_{0}}\left\|e_{v}\right\|_{L^{2}(\Omega)}^{2} .
\end{aligned}
$$


The third term of the left-hand side of (11) can be estimated easily as follows:

$$
\int_{0}^{T}\left\langle\nabla e_{\theta}, \nabla \zeta\right\rangle=\int_{0}^{t_{0}}\left\langle\nabla e_{\theta}, \int_{t}^{t_{0}} \nabla e_{\theta}\right\rangle=\frac{1}{2}\left\|\int_{0}^{t_{0}} \nabla e_{\theta}\right\|_{L^{2}(\Omega)}^{2} .
$$

Collecting all of the previous bounds yields

$$
\begin{aligned}
d_{1}\left\|e_{u}\right\|_{L^{2}\left(0, t_{0} ; L^{2}(\Omega)\right)}^{2} & +d_{2}\left\|e_{v}\right\|_{L^{2}\left(0, t_{0} ; L^{2}(\Omega)\right)}^{2}+\left\|\int_{0}^{t_{0}} \nabla e_{\theta}\right\|_{L^{2}(\Omega)}^{2} \\
& \leq C\left(\varepsilon^{1 / 2}+\left\|e_{z_{0}}\right\|_{L^{2}(\Omega)}^{2}\right)
\end{aligned}
$$

for all $t_{0} \in(0, T)$ and some positive constant $C$ independent of $\varepsilon$.

Let $\varphi$ be a function belonging to $H^{1}(\Omega)$, and let $t_{0}$ be an arbitrary point in $(0, T)$. The function $\chi_{\delta}=\chi_{\delta}(t)$ is defined as

$$
\chi_{\delta}(t)= \begin{cases}1 & t \in\left[0, t_{0}-\delta\right], \\ \left(t_{0}+\delta-t\right) / 2 \delta & t \in\left(t_{0}-\delta, t_{0}+\delta\right), \\ 0 & t \in\left[t_{0}+\delta, T\right] .\end{cases}
$$

The function $\chi_{\delta}$ converges in $L^{2}(0, T)$ to the characteristic function of $\left(0, t_{0}\right)$. Taking $\zeta(x, t)=\phi(x) \chi_{\delta}(t)$ in $(11)$, we obtain

$$
\frac{1}{2 \delta} \int_{t_{0}-\delta}^{t_{0}+\delta}\left\langle e_{z}, \varphi\right\rangle-\left\langle e_{z_{0}}, \varphi\right\rangle+\int_{0}^{T} \chi_{\delta}\left\langle\nabla e_{\theta}, \nabla \varphi\right\rangle=0 .
$$

Using the Lebesgue differentiation theorem and the Cauchy-Schwarz inequality, for a.e. $t_{0} \in(0, T)$, we have

$$
\begin{aligned}
\left|\left\langle e_{z}\left(t_{0}\right), \varphi\right\rangle\right| & \leq\left|\left\langle e_{z_{0}}, \varphi\right\rangle\right|+\left|\int_{0}^{t_{0}}\left\langle\nabla e_{\theta}, \nabla \varphi\right\rangle\right| \\
& \leq\left(\left\|e_{z_{0}}\right\|_{L^{2}(\Omega)}+\left\|\int_{0}^{t_{0}} \nabla e_{\theta}\right\|_{L^{2}(\Omega)}\right)\|\varphi\|_{H^{1}(\Omega)} .
\end{aligned}
$$

From the last inequality and (14), the desired estimate is obtained.

\section{A proof of Theorem 4}

In this section, a proof of Theorem 4 is presented. The strategy is the same as that for the proof of Theorem 3. The following lemmas hold for the functions $u_{\xi}^{\varepsilon}, v_{\xi}^{\varepsilon}$ and $w_{\xi}^{\varepsilon}$ in the same way as in Lemmas 6-8. 
Lemma 9 The following relations hold:

$$
0 \leq u_{\xi}^{\varepsilon} \leq M, \quad 0 \leq v_{\xi}^{\varepsilon} \leq M, \quad 0 \leq w_{\xi}^{\varepsilon} \leq \eta \quad \text { in } \quad Q .
$$

Lemma 10 There exists a positive constant $C$ independent of $\varepsilon$ and $\xi$ such that

$$
\int_{0}^{T}\left\{\left\langle u_{\xi}^{\varepsilon}, v_{\xi}^{\varepsilon}\right\rangle+\left\langle\eta-w_{\xi}^{\varepsilon}, u_{\xi}^{\varepsilon}\right\rangle+\left\langle v_{\xi}^{\varepsilon}, w_{\xi}^{\varepsilon}\right\rangle\right\} \leq C \varepsilon .
$$

Lemma 11 There exists a positive constant $C$ independent of $\varepsilon$ and $\xi$ such that

$$
d_{1}\left\|\nabla u_{\xi}^{\varepsilon}\right\|_{L^{2}(Q)}+d_{2}\left\|\nabla v_{\xi}^{\varepsilon}\right\|_{L^{2}(Q)}+\xi\left\|\nabla w_{\xi}^{\varepsilon}\right\|_{L^{2}(Q)} \leq C .
$$

From $(\mathrm{RD})_{\xi}$, we have

$$
\frac{\partial z_{\xi}^{\varepsilon}}{\partial t}=\Delta \theta_{\xi}^{\varepsilon}
$$

Multiplying this expression by a test function $\zeta \in \mathcal{K}$ and integrating by parts gives the following identity:

$$
\int_{0}^{T}\left\langle z_{\xi}^{\varepsilon}, \zeta_{t}\right\rangle+\left\langle z_{0}^{\varepsilon}, \zeta(\cdot, 0)\right\rangle=\int_{0}^{T}\left\langle\nabla \theta_{\xi}^{\varepsilon}, \nabla \zeta\right\rangle .
$$

After subtraction from (15) to (7), the following relation is obtained:

$$
-\int_{0}^{T}\left\langle e_{z}, \zeta_{t}\right\rangle-\left\langle e_{z_{0}}, \zeta(\cdot, 0)\right\rangle+\int_{0}^{T}\left\langle\nabla e_{\theta}, \nabla \zeta\right\rangle=0
$$

for all $\zeta \in \mathcal{K}$. Here, the errors are defined as follows:

$$
e_{z}:=z-z_{\xi}^{\varepsilon}, \quad e_{\theta}:=\theta-\theta_{\xi}^{\varepsilon}, \quad e_{z_{0}}:=z_{0}-z_{0}^{\varepsilon} .
$$

Now, we are ready to prove Theorem 4 .

Proof of Theorem 4. The strategy is the same as that in the proof of Theorem 3. To this end, we take

$$
\zeta(x, t)= \begin{cases}\int_{t}^{t_{0}} e_{\theta}(x, s) d s & \text { if } 0 \leq t<t_{0} \\ 0 & \text { if } t_{0} \leq t \leq T\end{cases}
$$

for fixed $t_{0} \in(0, T]$ in $(16)$.

The term $\left\langle e_{z}, e_{\theta}\right\rangle$ is then estimated. For a.e. $(x, t) \in Q$,

$$
\left\langle e_{z}, e_{\theta}\right\rangle=\left\langle e_{z}, \theta-\beta_{\xi}(z)\right\rangle+\left\langle e_{z}, \beta_{\xi}(z)-\beta_{\xi}\left(z_{\xi}^{\varepsilon}\right)\right\rangle+\left\langle e_{z}, \beta_{\xi}\left(z_{\xi}^{\varepsilon}\right)-\theta_{\xi}^{\varepsilon}\right\rangle .
$$


Using the elementary relation (13), the first term is estimated as follows:

$$
\begin{aligned}
\left\langle e_{z}, \theta-\beta_{\xi}(z)\right\rangle= & \left\langle e_{u}-e_{v}+e_{w}, \beta(z)-\beta_{\xi}(z)\right\rangle \\
\geq & -\frac{d_{1}}{8}\left\|e_{u}\right\|_{L^{2}(\Omega)}^{2}-\frac{d_{2}}{8}\left\|e_{v}\right\|_{L^{2}(\Omega)}^{2}-\frac{\xi}{8}\left\|e_{w}\right\|_{L^{2}(\Omega)}^{2} \\
& -\left(\frac{2}{d_{1}}+\frac{2}{d_{2}}+\frac{2}{\xi}\right)\left\|\beta(z)-\beta_{\xi}(z)\right\|_{L^{2}(\Omega)}^{2},
\end{aligned}
$$

where $e_{u}:=u-u_{\xi}^{\varepsilon}, e_{v}:=v-v_{\xi}^{\varepsilon}$ and $e_{w}:=w-w_{\xi}^{\varepsilon}$.

The last term of (17) can be estimated in a similar manner:

$$
\begin{aligned}
\left\langle e_{z}, \beta_{\xi}\left(z_{\xi}^{\varepsilon}\right)-\theta_{\xi}^{\varepsilon}\right\rangle \geq & -\frac{d_{1}}{16}\left\|e_{u}\right\|_{L^{2}(\Omega)}^{2}-\frac{d_{2}}{16}\left\|e_{v}\right\|_{L^{2}(\Omega)}^{2}-\frac{\xi}{16}\left\|e_{w}\right\|_{L^{2}(\Omega)}^{2} \\
& -\left(\frac{4}{d_{1}}+\frac{4}{d_{2}}+\frac{4}{\xi}\right)\left\|\beta_{\xi}\left(z_{\xi}^{\varepsilon}\right)-\theta_{\xi}^{\varepsilon}\right\|_{L^{2}(\Omega)}^{2} .
\end{aligned}
$$

It follows from the property of $\phi_{1}^{\xi}$ and the triangle inequality that

$$
\begin{aligned}
& \left\langle e_{z}, \phi_{1}^{\xi}(z)-\phi_{1}^{\xi}\left(z_{\xi}^{\varepsilon}\right)\right\rangle \geq\left\|\phi_{1}^{\xi}(z)-\phi_{1}^{\xi}\left(z_{\xi}^{\varepsilon}\right)\right\|_{L^{2}(\Omega)}^{2} \\
& \quad \geq \frac{1}{2}\left\|\phi_{1}(z)-\phi_{1}^{\xi}\left(z_{\xi}^{\varepsilon}\right)\right\|_{L^{2}(\Omega)}^{2}-\left\|\phi_{1}(z)-\phi_{1}^{\xi}(z)\right\|_{L^{2}(\Omega)}^{2} \\
& \quad \geq \frac{1}{4}\left\|e_{u}\right\|_{L^{2}(\Omega)}^{2}-\frac{1}{2}\left\|\phi_{1}^{\xi}\left(z_{\xi}^{\varepsilon}\right)-u_{\xi}^{\varepsilon}\right\|_{L^{2}(\Omega)}^{2}-\left\|\phi_{1}(z)-\phi_{1}^{\xi}(z)\right\|_{L^{2}(\Omega)}^{2} .
\end{aligned}
$$

Similarly, we obtain

$$
-\left\langle e_{z}, \phi_{2}(z)-\phi_{2}\left(z_{\xi}^{\varepsilon}\right)\right\rangle \geq \frac{1}{2}\left\|e_{v}\right\|_{L^{2}(\Omega)}^{2}-\left\|\phi_{2}\left(z_{\xi}^{\varepsilon}\right)-v_{\xi}^{\varepsilon}\right\|_{L^{2}(\Omega)}^{2}
$$

and

$$
\begin{aligned}
& \left\langle e_{w}, \phi_{3}^{\xi}(z)-\phi_{3}^{\xi}\left(z_{\xi}^{\varepsilon}\right)\right\rangle \\
& \quad \geq \frac{1}{4}\left\|e_{w}\right\|_{L^{2}(\Omega)}^{2}-\frac{1}{2}\left\|\phi_{3}^{\xi}\left(z_{\xi}^{\varepsilon}\right)-w_{\xi}^{\varepsilon}\right\|_{L^{2}(\Omega)}^{2}-\left\|\phi_{3}(z)-\phi_{3}^{\xi}(z)\right\|_{L^{2}(\Omega)}^{2} .
\end{aligned}
$$

Therefore, the second term of (17) can be estimated as follows:

$$
\begin{aligned}
& \left\langle e_{z}, \beta_{\xi}(z)-\beta_{\xi}\left(z_{\xi}^{\varepsilon}\right)\right\rangle \geq \frac{d_{1}}{4}\left\|e_{u}\right\|_{L^{2}(\Omega)}^{2}+\frac{d_{2}}{2}\left\|e_{v}\right\|_{L^{2}(\Omega)}^{2}+\frac{\xi}{4}\left\|e_{w}\right\|_{L^{2}(\Omega)}^{2} \\
& \quad-\frac{d_{1}}{2}\left\|\phi_{1}^{\xi}\left(z_{\xi}^{\varepsilon}\right)-u_{\xi}^{\varepsilon}\right\|_{L^{2}(\Omega)}^{2}-d_{2}\left\|\phi_{2}\left(z_{\xi}^{\varepsilon}\right)-v_{\xi}^{\varepsilon}\right\|_{L^{2}(\Omega)}^{2}-\frac{\xi}{2}\left\|\phi_{3}^{\xi}\left(z_{\xi}^{\varepsilon}\right)-w_{\xi}^{\varepsilon}\right\|_{L^{2}(\Omega)}^{2} \\
& \quad-d_{1}\left\|\phi_{1}(z)-\phi_{1}^{\xi}(z)\right\|_{L^{2}(\Omega)}^{2}-\xi\left\|\phi_{3}(z)-\phi_{3}^{\xi}(z)\right\|_{L^{2}(\Omega)}^{2} .
\end{aligned}
$$


It follows from the property of $\phi_{i}^{\xi}(i=1,3)$ and the property $\beta(z)=\beta_{\xi}(z)$ if $(x, t) \notin A_{\xi}(z)$ that

$$
\begin{gathered}
\left\|\phi_{1}(z)-\phi_{1}^{\xi}(z)\right\|_{L^{2}(\Omega)}^{2}+\left\|\phi_{3}(z)-\phi_{3}^{\xi}(z)\right\|_{L^{2}(\Omega)}^{2} \leq|\Omega| \xi^{2}, \\
\left\|\beta(z)-\beta_{\xi}(z)\right\|_{L^{2}(\Omega)}^{2} \leq \xi^{2}\left|A_{\xi}(z)\right| .
\end{gathered}
$$

Using Lemma 10 and the inequalities for $u, v \geq 0$ and $w \in[0, \eta]$ :

$$
\left\{\begin{array}{l}
\left|u-\phi_{1}^{\xi}(u-v+w)\right|^{2} \leq u v+(\eta-w) u \\
\left|v-\phi_{2}(u-v+w)\right|^{2} \leq u v+v w \\
\left|w-\phi_{3}^{\xi}(u-v+w)\right|^{2} \leq v w+(\eta-w) u
\end{array}\right.
$$

we obtain

$$
\begin{array}{r}
\left\|\phi_{1}^{\xi}\left(z_{\xi}^{\varepsilon}\right)-u_{\xi}^{\varepsilon}\right\|_{L^{2}(Q)}^{2}+\left\|\phi_{2}\left(z_{\xi}^{\varepsilon}\right)-v_{\xi}^{\varepsilon}\right\|_{L^{2}(Q)}^{2}+\left\|\phi_{3}^{\xi}\left(z_{\xi}^{\varepsilon}\right)-w_{\xi}^{\varepsilon}\right\|_{L^{2}(Q)}^{2} \\
+\left\|\beta_{\xi}\left(z_{\xi}^{\varepsilon}\right)-\theta_{\xi}^{\varepsilon}\right\|_{L^{2}(Q)}^{2} \leq C \varepsilon
\end{array}
$$

for some positive constant $C$ independent of $\varepsilon$ and $\xi$.

The other terms of (16) can be estimated in the same manner as in Theorem 3.

Collecting these bounds yields

$$
\begin{aligned}
d_{1}\left\|e_{u}\right\|_{L^{2}\left(0, t_{0} ; L^{2}(\Omega)\right)}^{2} & +d_{2}\left\|e_{v}\right\|_{L^{2}\left(0, t_{0} ; L^{2}(\Omega)\right)}^{2}+\xi\left\|e_{w}\right\|_{L^{2}\left(0, t_{0} ; L^{2}(\Omega)\right)}^{2}+\left\|\int_{0}^{t_{0}} \nabla e_{\theta}\right\|_{L^{2}(\Omega)}^{2} \\
& \leq C\left(\xi\left|A_{\xi}(z)\right|+\xi^{2}+\frac{\varepsilon}{\xi}+\sigma(\varepsilon, \xi)\right)
\end{aligned}
$$

for all $t_{0} \in(0, T)$.

The assertion can be proven analogously as Theorem 3 .

\section{Concluding remarks}

A new reaction-diffusion system approximation to the classical Stefan problem with sufficiently small parameters $\varepsilon$ and $\xi$ was proposed from a regularization point of view. The rates of convergence with respect to $\varepsilon$ and $\xi$ have been investigated theoretically. Numerical experiments indicate that the present results for the convergence rates can be improved [13]. The optimal rate of convergence, including the convergence of the interface, should be investigated. 


\section{Acknowledgements}

This study was supported in part by the Japan Society for the Promotion of Science and by the Kyushu University 21st Century COE Program, Development of Dynamic Mathematics with High Functionality, of the Ministry of Education, Culture, Sports, Science and Technology of Japan.

\section{References}

[1] G. Beckett, J. A. Mackenzie and M. L. Robertson, A moving mesh finite element method for the solution of two-dimensional Stefan problems, J. Comp. Phys. 168 (2001) 500-518.

[2] J. Crank, Free and moving boundary problems (Clarendon Press, Oxford, 1984).

[3] E. C. M. Grooks, E. N. Dancer, D. Hilhorst, M. Mimura and H. Ninomiya, Spatial segregation limit of a competition-diffusion system with Dirichlet boundary conditions, Nonlinear Analysis: Real World Applications 5 (2004) $645-665$.

[4] E. N. Dancer, D. Hilhorst, M. Mimura and L. A. Peletier, Spatial segregation limit of a competition-diffusion system, Euro. J. Appl. Math. 10 (1999) 97-115.

[5] A. Friedman, The Stefan problem in several space variables, Trans. Amer. Math. Soc. 133 (1968) 51-87.

[6] C. Grossmann and A. Noack, Smoothing and Rothe's method for Stefan problems in enthalpy form, J. Comp. Appl. Math. 138 (2002) 347-366.

[7] D. Hilhorst, M. Iida, M. Mimura and H. Ninomiya, A competition-diffusion system approximation to the classical two-phase Stefan problem, Japan J. Indust. Appl. Math. 18 (2001) 161-180.

[8] D. Hilhorst, M. Iida, M. Mimura and H. Ninomiya, A reaction-diffusion system approximation to the classical two-phase Stefan problem, Nonlinear Analysis 47 (2001) 801-812.

[9] D. Hilhorst, M. Mimura and R. Schätzle, Vanishing latent heat limit in a Stefanlike problem arising in biology, Nonlinear Analysis 4 (2003) 261-285.

[10] O. A. Ladyženskaja, V. A. Solonnikov and N. N. Ural'ceva, Linear and quasilinear equations of parabolic type (Trans. Math. Monographs 23, American Mathematical Society, Providence, Rhode Island, 1968).

[11] A. Lunardi, Analytic semigroupes and regularity in parabolic problems (Progress in nonlinear differential equations and their applications, 16, Birkhäuser, Basel·Boston·Berlin, 1995). 
[12] A. M. Meirmanov, The Stefan problem (Walter de Gruyter, Berlin, 1992).

[13] H. Murakawa, On reaction-diffusion system approximations to the classical Stefan problems, preprint.

[14] R. H. Nochetto, Error estimates for two-phase Stefan problems in several space variables, I: linear boundary conditions, Calcolo 22 (1985) 457-499.

[15] R. H. Nochetto, A class of non-degenerate two-phase Stefan problems in several space variables, Comm. Partial Differential Equations 12 (1987) 111-138. 


\section{List of MHF Preprint Series, Kyushu University 21st Century COE Program Development of Dynamic Mathematics with High Functionality}

\section{MHF2003-1 Mitsuhiro T. NAKAO, Kouji HASHIMOTO \& Yoshitaka WATANABE}

A numerical method to verify the invertibility of linear elliptic operators with applications to nonlinear problems

MHF2003-2 Masahisa TABATA \& Daisuke TAGAMI

Error estimates of finite element methods for nonstationary thermal convection problems with temperature-dependent coefficients

MHF2003-3 Tomohiro ANDO, Sadanori KONISHI \& Seiya IMOTO

Adaptive learning machines for nonlinear classification and Bayesian information criteria

MHF2003-4 Kazuhiro YOKOYAMA

On systems of algebraic equations with parametric exponents

MHF2003-5 Masao ISHIKAWA \& Masato WAKAYAMA

Applications of Minor Summation Formulas III, Plücker relations, Lattice paths and Pfaffian identities

MHF2003-6 Atsushi SUZUKI \& Masahisa TABATA

Finite element matrices in congruent subdomains and their effective use for large-scale computations

MHF2003-7 Setsuo TANIGUCHI

Stochastic oscillatory integrals - asymptotic and exact expressions for quadratic phase functions -

MHF2003-8 Shoki MIYAMOTO \& Atsushi YOSHIKAWA

Computable sequences in the Sobolev spaces

MHF2003-9 Toru FUJII \& Takashi YANAGAWA

Wavelet based estimate for non-linear and non-stationary auto-regressive model

MHF2003-10 Atsushi YOSHIKAWA

Maple and wave-front tracking - an experiment

MHF2003-11 Masanobu KANEKO

On the local factor of the zeta function of quadratic orders

MHF2003-12 Hidefumi KAWASAKI

Conjugate-set game for a nonlinear programming problem 
MHF2004-1 Koji YONEMOTO \& Takashi YANAGAWA

Estimating the Lyapunov exponent from chaotic time series with dynamic noise

MHF2004-2 Rui YAMAGUCHI, Eiko TSUCHIYA \& Tomoyuki HIGUCHI

State space modeling approach to decompose daily sales of a restaurant into time-dependent multi-factors

MHF2004-3 Kenji KAJIWARA, Tetsu MASUDA, Masatoshi NOUMI, Yasuhiro OHTA \& Yasuhiko YAMADA

Cubic pencils and Painlevé Hamiltonians

MHF2004-4 Atsushi KAWAGUCHI, Koji YONEMOTO \& Takashi YANAGAWA

Estimating the correlation dimension from a chaotic system with dynamic noise

MHF2004-5 Atsushi KAWAGUCHI, Kentarou KITAMURA, Koji YONEMOTO, Takashi YANAGAWA \& Kiyofumi YUMOTO

Detection of auroral breakups using the correlation dimension

MHF2004-6 Ryo IKOTA, Masayasu MIMURA \& Tatsuyuki NAKAKI

A methodology for numerical simulations to a singular limit

MHF2004-7 Ryo IKOTA \& Eiji YANAGIDA

Stability of stationary interfaces of binary-tree type

MHF2004-8 Yuko ARAKI, Sadanori KONISHI \& Seiya IMOTO

Functional discriminant analysis for gene expression data via radial basis expansion

MHF2004-9 Kenji KAJIWARA, Tetsu MASUDA, Masatoshi NOUMI, Yasuhiro OHTA \& Yasuhiko YAMADA

Hypergeometric solutions to the $q$ - Painlevé equations

MHF2004-10 Raimundas VIDŪNAS

Expressions for values of the gamma function

MHF2004-11 Raimundas VIDŪNAS

Transformations of Gauss hypergeometric functions

MHF2004-12 Koji NAKAGAWA \& Masakazu SUZUKI

Mathematical knowledge browser

MHF2004-13 Ken-ichi MARUNO, Wen-Xiu MA \& Masayuki OIKAWA

Generalized Casorati determinant and Positon-Negaton-Type solutions of the Toda lattice equation

MHF2004-14 Nalini JOSHI, Kenji KAJIWARA \& Marta MAZZOCCO

Generating function associated with the determinant formula for the solutions of the Painlevé II equation 
MHF2004-15 Kouji HASHIMOTO, Ryohei ABE, Mitsuhiro T. NAKAO \& Yoshitaka WATANABE

Numerical verification methods of solutions for nonlinear singularly perturbed problem

MHF2004-16 Ken-ichi MARUNO \& Gino BIONDINI

Resonance and web structure in discrete soliton systems: the two-dimensional Toda lattice and its fully discrete and ultra-discrete versions

MHF2004-17 Ryuei NISHII \& Shinto EGUCHI

Supervised image classification in Markov random field models with Jeffreys divergence

MHF2004-18 Kouji HASHIMOTO, Kenta KOBAYASHI \& Mitsuhiro T. NAKAO

Numerical verification methods of solutions for the free boundary problem

MHF2004-19 Hiroki MASUDA

Ergodicity and exponential $\beta$-mixing bounds for a strong solution of Lévydriven stochastic differential equations

MHF2004-20 Setsuo TANIGUCHI

The Brownian sheet and the reflectionless potentials

MHF2004-21 Ryuei NISHII \& Shinto EGUCHI

Supervised image classification based on AdaBoost with contextual weak classifiers

MHF2004-22 Hideki KOSAKI

On intersections of domains of unbounded positive operators

MHF2004-23 Masahisa TABATA \& Shoichi FUJIMA

Robustness of a characteristic finite element scheme of second order in time increment

MHF2004-24 Ken-ichi MARUNO, Adrian ANKIEWICZ \& Nail AKHMEDIEV

Dissipative solitons of the discrete complex cubic-quintic Ginzburg-Landau equation

MHF2004-25 Raimundas VIDŪNAS

Degenerate Gauss hypergeometric functions

MHF2004-26 Ryo IKOTA

The boundedness of propagation speeds of disturbances for reaction-diffusion systems

MHF2004-27 Ryusuke KON

Convex dominates concave: an exclusion principle in discrete-time Kolmogorov systems 
MHF2004-28 Ryusuke KON

Multiple attractors in host-parasitoid interactions: coexistence and extinction

MHF2004-29 Kentaro IHARA, Masanobu KANEKO \& Don ZAGIER

Derivation and double shuffle relations for multiple zeta values

MHF2004-30 Shuichi INOKUCHI \& Yoshihiro MIZOGUCHI

Generalized partitioned quantum cellular automata and quantization of classical CA

MHF2005-1 Hideki KOSAKI

Matrix trace inequalities related to uncertainty principle

MHF2005-2 Masahisa TABATA

Discrepancy between theory and real computation on the stability of some finite element schemes

MHF2005-3 Yuko ARAKI \& Sadanori KONISHI

Functional regression modeling via regularized basis expansions and model selection

MHF2005-4 Yuko ARAKI \& Sadanori KONISHI

Functional discriminant analysis via regularized basis expansions

MHF2005-5 Kenji KAJIWARA, Tetsu MASUDA, Masatoshi NOUMI, Yasuhiro OHTA \& Yasuhiko YAMADA

Point configurations, Cremona transformations and the elliptic difference Painlevé equations

MHF2005-6 Kenji KAJIWARA, Tetsu MASUDA, Masatoshi NOUMI, Yasuhiro OHTA \& Yasuhiko YAMADA

Construction of hypergeometric solutions to the $q$ - Painlevé equations

MHF2005-7 Hiroki MASUDA

Simple estimators for non-linear Markovian trend from sampled data:

I. ergodic cases

MHF2005-8 Hiroki MASUDA \& Nakahiro YOSHIDA

Edgeworth expansion for a class of Ornstein-Uhlenbeck-based models

MHF2005-9 Masayuki UCHIDA

Approximate martingale estimating functions under small perturbations of dynamical systems

MHF2005-10 Ryo MATSUZAKI \& Masayuki UCHIDA

One-step estimators for diffusion processes with small dispersion parameters from discrete observations

MHF2005-11 Junichi MATSUKUBO, Ryo MATSUZAKI \& Masayuki UCHIDA

Estimation for a discretely observed small diffusion process with a linear drift 
MHF2005-12 Masayuki UCHIDA \& Nakahiro YOSHIDA

AIC for ergodic diffusion processes from discrete observations

MHF2005-13 Hiromichi GOTO \& Kenji KAJIWARA

Generating function related to the Okamoto polynomials for the Painlevé IV equation

MHF2005-14 Masato KIMURA \& Shin-ichi NAGATA

Precise asymptotic behaviour of the first eigenvalue of Sturm-Liouville problems with large drift

MHF2005-15 Daisuke TAGAMI \& Masahisa TABATA

Numerical computations of a melting glass convection in the furnace

MHF2005-16 Raimundas VIDŪNAS

Normalized Leonard pairs and Askey-Wilson relations

MHF2005-17 Raimundas VIDŪNAS

Askey-Wilson relations and Leonard pairs

MHF2005-18 Kenji KAJIWARA \& Atsushi MUKAIHIRA

Soliton solutions for the non-autonomous discrete-time Toda lattice equation

MHF2005-19 Yuu HARIYA

Construction of Gibbs measures for 1-dimensional continuum fields

MHF2005-20 Yuu HARIYA

Integration by parts formulae for the Wiener measure restricted to subsets in $\mathbb{R}^{d}$

MHF2005-21 Yuu HARIYA

A time-change approach to Kotani's extension of Yor's formula

MHF2005-22 Tadahisa FUNAKI, Yuu HARIYA \& Mark YOR

Wiener integrals for centered powers of Bessel processes, I

MHF2005-23 Masahisa TABATA \& Satoshi KAIZU

Finite element schemes for two-fluids flow problems

MHF2005-24 Ken-ichi MARUNO \& Yasuhiro OHTA

Determinant form of dark soliton solutions of the discrete nonlinear Schrödinger equation

MHF2005-25 Alexander V. KITAEV \& Raimundas VIDŪNAS

Quadratic transformations of the sixth Painlevé equation

MHF2005-26 Toru FUJII \& Sadanori KONISHI

Nonlinear regression modeling via regularized wavelets and smoothing parameter selection 
MHF2005-27 Shuichi INOKUCHI, Kazumasa HONDA, Hyen Yeal LEE, Tatsuro SATO, Yoshihiro MIZOGUCHI \& Yasuo KAWAHARA

On reversible cellular automata with finite cell array

MHF2005-28 Toru KOMATSU

Cyclic cubic field with explicit Artin symbols

MHF2005-29 Mitsuhiro T. NAKAO, Kouji HASHIMOTO \& Kaori NAGATOU

A computational approach to constructive a priori and a posteriori error estimates for finite element approximations of bi-harmonic problems

MHF2005-30 Kaori NAGATOU, Kouji HASHIMOTO \& Mitsuhiro T. NAKAO

Numerical verification of stationary solutions for Navier-Stokes problems

MHF2005-31 Hidefumi KAWASAKI

A duality theorem for a three-phase partition problem

MHF2005-32 Hidefumi KAWASAKI

A duality theorem based on triangles separating three convex sets

MHF2005-33 Takeaki FUCHIKAMI \& Hidefumi KAWASAKI

An explicit formula of the Shapley value for a cooperative game induced from the conjugate point

MHF2005-34 Hideki MURAKAWA

A regularization of a reaction-diffusion system approximation to the two-phase

Stefan problem 